\title{
Atomistic Modeling Of Co Growth On Cu(111)
}

\section{Joseph Khali1 ${ }^{1}$, Guillermo Bozzolo ${ }^{1}$, Daniel Farías², A.L. Vázquez de Parga ${ }^{2}$, J.J. de Miguel $^{2}$ and R. Miranda ${ }^{2}$}

${ }^{1}$ Ohio Aerospace Institute, 22800 Cedar Point Road, Cleveland, OH 44142, USA and National Aeronautics and Space Administration, Glenn Research Center, Cleveland, OH, 44135, USA

${ }^{2}$ Departamento de Física de la Materia Condensada, Universidad Autónoma de Madrid, Madrid, Spain.

\begin{abstract}
The BFS method for alloys is applied to the study of Co growth on $\mathrm{Cu}(111)$. The parameterization of the $\mathrm{Co}-\mathrm{Cu}$ system is obtained from first-principles calculations, and tested against known experimental features for low coverage $\mathrm{Co}$ deposition on $\mathrm{Cu}(100)$ and $\mathrm{Cu}(111)$. Atomistic simulations are performed to investigate the behavior of $\mathrm{Co}$ on $\mathrm{Cu}(111)$ as a function of coverage.
\end{abstract}

\section{INTRODUCTION}

Recent experimental analysis of $\mathrm{Co}$ growth on $\mathrm{Cu}(111)$ has raised interesting questions regarding the low coverage regime and the identification of the driving mechanisms that lead to the observed structures. The understanding of such phenomena would allow for controlling the structure and morphology of the growing films to achieve the desired properties. Some of the observed features include triangular-shaped islands with a height of two layers above the surface, decoration of the $\mathrm{Cu}$ step edges with mixed $\mathrm{Co}$ and $\mathrm{Cu}$ clusters, and monatomic-deep pools of vacancies in the surface [1]. We present preliminary results of a modeling effort based on the BFS method for alloys [2], meant to elucidate the main characteristics of the early growth stages of $\mathrm{Co}$ films on $\mathrm{Cu}(111)$. Analytical calculations for the determination of the surface structure are supplemented with temperature-dependent Monte Carlo simulations. After testing the validity of the BFS parameterization (done with first-principles methods) by reproducing basic known features of the system, the methodology is applied to the study of multi-layer islands, their shape, composition and orientation.

\section{THE BFS METHOD}

The BFS method [2] is based on the concept that the energy of formation of a given atomic configuration is the superposition of the individual atomic contributions $\Delta H=\Sigma \varepsilon_{i}$. Each contribution $\varepsilon_{i}$ is the sum of two terms: a strain energy, $\varepsilon_{i}^{S}$, computed in the actual lattice as if every neighbor of the atom $i$ was of the same atomic species $i$, and a chemical energy, $\varepsilon_{i}^{C}$, computed as if every neighbor of the atom $i$ was in an equilibrium lattice site of a crystal of species $i$, but retaining its actual chemical identity. The computation of $\varepsilon_{i}^{S}$, using Equivalent Crystal Theory [3], involves three pure element properties for atoms of species $i$ : cohesive energy, lattice parameter and bulk modulus. The chemical energy, $\varepsilon_{i}^{C}$, includes two BFS concentration-dependent perturbative parameters $\left(\Delta_{\mathrm{CoCu}}\right.$ and $\left.\Delta_{\mathrm{CuCo}}\right)$ [2]. A reference chemical 


\begin{tabular}{|c|c|c|c|c|c|c|c|}
\hline & $\begin{array}{c}\text { Lattice } \\
\text { parameter }(\AA)\end{array}$ & $\begin{array}{c}\text { Cohesive } \\
\text { energy (eV) }\end{array}$ & $\begin{array}{c}\text { Bulk } \\
\text { modulus }(\mathrm{GPa})\end{array}$ & $\mathrm{p}$ & $\alpha\left(\AA^{-1}\right)$ & $l\left(\AA^{-1}\right)$ & $\lambda(\AA)$ \\
\hline $\mathrm{Co}$ & 3.45803 & 5.56374 & 255.21 & 6 & 3.01887 & 0.26166 & 0.73527 \\
\hline $\mathrm{Cu}$ & 3.63255 & 3.55808 & 141.79 & 6 & 2.87172 & 0.27390 & 0.76966 \\
\hline
\end{tabular}

Table I: LAPW [4] results for the lattice parameter, cohesive energy, and bulk modulus for the fcc phases of Co and $\mathrm{Cu}$, and the resulting Equivalent Crystal Theory (ECT) [3] parameters p, $\alpha, l$ and $\lambda$ (see text and Ref. 2).

energy, $\varepsilon_{i}^{(0)}$, is also included to insure a complete decoupling of structural and chemical features. From the theoretical standpoint, the immiscibility of $\mathrm{Co}$ and $\mathrm{Cu}$ in the bulk presents a challenge, due to the lack of accurate experimental evidence that would help validate the parameterization used. However, although these binary phases do not exist, their properties can be readily computed via first-principles methods. Therefore, in this work, all the necessary parameters were determined using the linearized augmented plane wave method (LAPW) [4], by computing the equilibrium properties of $\mathrm{Co}$ and $\mathrm{Cu}$ in the fcc phase, as well as the equilibrium properties of $\mathrm{Co}_{3} \mathrm{Cu}\left(\mathrm{Ll}_{2}\right), \mathrm{CoCu}\left(\mathrm{Ll}_{1}\right)$ and $\mathrm{CoCu}_{3}\left(\mathrm{Ll}_{2}\right)$ ordered alloys. Moreover, this calculational scheme enables an accurate determination of the concentration-dependence of the BFS parameters $\Delta$. The functions used in this work are given by $\Delta_{C_{0} c_{u}}\left(x_{C_{0}}\right)=0.045003 \ln \left(x_{C_{0}}\right)-0.047823$ and $\Delta_{\mathrm{CuCO}_{\mathrm{O}}}\left(x_{\mathrm{C}_{\mathrm{H}}}\right)=-0.210113 x_{\mathrm{C}_{11}}+0.410351$, where $x_{\mathrm{C}^{\prime}}\left(x_{\mathrm{C}_{11}}\right)$ denotes the fraction of nearest-neighbors of a given $\mathrm{Cu}(\mathrm{Co})$ atom that are $\mathrm{Co}(\mathrm{Cu})$ atoms. Finally, the strain and chemical energies are linked with a coupling function $g$, which ensures the correct volume dependence of the BFS chemical energy contribution. Therefore, the contribution of atom $i$ to the energy of formation of the system is given by

$$
\varepsilon_{i}=\varepsilon_{i}^{S}+g_{i}\left(\varepsilon_{i}^{C}-\varepsilon_{i}^{C^{C}}\right)
$$

Table I lists the necessary parameters for applying the BFS method to the Co-Cu system. The procedure used to determine the $\Delta(x)$ functions exceeds the scope of this paper and will be published elsewhere. We refer the reader to Ref. 2 for a detailed discussion of the BFS method, its definitions, operational equations and their implementation.

\section{RESULTS AND DISCUSSION}

Obtaining atomically sharp interfaces between different layers is one of the main problems in the preparation of thin films or superlattices. Intermixing is a common phenomenon, even between elements that are immiscible in the bulk. A typical example is shown in Fig. 1, where the STM image shows a $\mathrm{Cu}(111)$ surface after growing $0.3 \mathrm{ML}$ of $\mathrm{Co}$ at room temperature. A detailed study of the image reveals the presence of multilayer, mixed composition islands, with two or more atomic layers over the $\mathrm{Cu}(111)$ surface [5]. It is also found that there are pools of single-atomic-depth vacancies between the islands, absent in clean $\mathrm{Cu}(111)$ surfaces. Other remarkable features include the fact that the total volume of the islands exceeds the amount of Co deposited, as well as evidence for the segregation of $\mathrm{Cu}$ to the island surface, as confirmed by ion scattering spectroscopy [6] and chemical titration [5] experiments. These features are highlighted in the inset in Fig. 1, where an enlarged view of one of the islands clearly shows changes in the right side corners of the islands. STM experiments [7] also suggest that the islands include a subsurface Co layer. In addition, theoretical calculations using empirical interatomic potentials indicate that two-dimensional $\mathrm{Co}$ islands become unstable above a certain critical size and "explode" upwards, dragging $\mathrm{Cu}$ atoms from the surface layer forming mixed- 


\begin{tabular}{lcc}
\hline & $\mathrm{Cu}(100)$ & $\mathrm{Cu}(111)$ \\
\hline $\mathrm{Co}(\mathrm{O})$ & 2.525 & 3.356 \\
$\mathrm{Co}(\mathrm{S}) \mathrm{Cu}(\mathrm{O})_{1}$ & 2.403 & 3.176 \\
$\mathrm{Co}(\mathrm{S}) \mathrm{Cu}(\mathrm{O})_{\mathrm{r}}$ & 2.359 & 3.081 \\
$\mathrm{Co}(1 \mathrm{~b})$ & 2.632 & 3.360 \\
$\mathrm{Co}(2 \mathrm{~b})$ & 2.696 & 3.415 \\
\hline
\end{tabular}

Table II: BFS total energy difference (in $\mathrm{eV}$ ) between different configurations including a $\mathrm{Co}$ atom and $\mathrm{a} \mathrm{Cu}(100)$ or a $\mathrm{Cu}(111)$ substrate and the corresponding clean surfaces: (a) a Co atom in an overlayer site $(\mathrm{Co}(\mathrm{O}))$, a $\mathrm{Co}$ atom in a surface site with the ejected $\mathrm{Cu}$ atom in an overlayer site at (b) nearest-neighbor distance $\left(\mathrm{Co}(\mathrm{S}) \mathrm{Cu}(\mathrm{O})_{1}\right)$ or $(\mathrm{c})$ far from the $\mathrm{Co}(\mathrm{S})$ atom $\left(\mathrm{Co}(\mathrm{S}) \mathrm{Cu}(\mathrm{O})_{\mathrm{f}}\right)$, and with the $\mathrm{Co}$ atom $(\mathrm{d})$ one and (e) two planes below the surface.

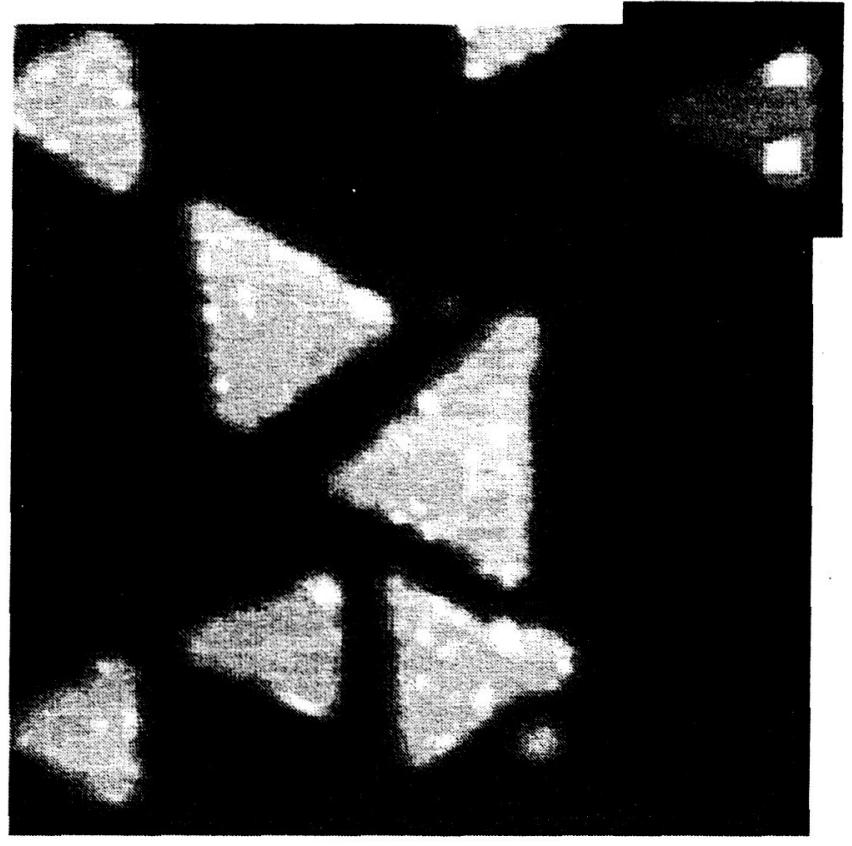

Figure 1: A $50 \times 50 \mathrm{~nm}$ STM image of $0.3 \mathrm{ML}$ Co deposited at room temperature on $\mathrm{Cu}(111)$. The islands that appear on the surface are $4.1 \mathrm{~nm}$ high over the $\mathrm{Cu}(111)$ surface, corresponding to a double layer of Co. The inset shows an enlarged view of one of the islands, highlighting the fact that the composition of the islands is not homogeneous. The black hexagons correspond to vacancy pools in the $\mathrm{Cu}$ surface.

composition islands several layers high [8].

From a modeling standpoint, the growth pattern of $\mathrm{Co}$ on $\mathrm{Cu}$ with increasing Co coverage is related to the ability of $\mathrm{Co}$ to interdiffuse in the $\mathrm{Cu}$ substrate. A simple atom-by-atom $\mathrm{BFS}$ calculation of several configurations including one $\mathrm{Co}$ atom deposited on a $\mathrm{Cu}(111)$ substrate indicates that an exchange mechanism by which $\mathrm{Co}$ atoms substitute for $\mathrm{Cu}$ surface atoms is favored. The calculation also shows that a lower energy state is reached if the $\mathrm{Cu}$ atom is allowed to occupy an overlayer site away from the inserted $\mathrm{Co}$ atom $(\mathrm{Co}(\mathrm{S}))$. Table 2 lists the energy differences between a pure $\mathrm{Cu}(100)$ or $\mathrm{Cu}(111)$ substrate and a state where (a) the Co atom stays in an overlayer site $(\mathrm{Co}(\mathrm{O}))$, (b) Co replaces a $\mathrm{Cu}$ surface atom which stays in a nearest-neighbor overlayer site $\left(\mathrm{Co}(\mathrm{S}) \mathrm{Cu}(\mathrm{O})_{1}\right)$, (c) far from the inserted $\mathrm{Co}$ atom $\left(\mathrm{Co}(\mathrm{S}) \mathrm{Cu}(\mathrm{O})_{\mathrm{f}}\right)$, (d) in the first $(\mathrm{Co}(\mathrm{lb}))$ and $(\mathrm{e})$ in the second $(\mathrm{Co}(2 \mathrm{~b}))$ layer below the surface. The noticeable energy gain in performing the exchange from $\mathrm{Co}(\mathrm{O})$ to $\mathrm{Co}(\mathrm{S}) \mathrm{Cu}(\mathrm{O})$ is a result of the lower surface energy of $\mathrm{Cu}$ than that of $\mathrm{Co}$. For both surfaces, the $\mathrm{Co}(\mathrm{S}) \mathrm{Cu}(\mathrm{O})_{\mathrm{f}}$ is the configuration with lowest energy indicating that, in principle, Co atoms would not interdiffuse beyond the surface plane. A more detailed discussion concerning the different BFS energy contributions and their consequences in the deposition of $\mathrm{Co}$ on $\mathrm{Cu}$ will be presented elsewhere.

Beyond the simple case of one adatom, Monte Carlo simulations provide useful information on the resulting behavior for higher coverages. In these simulations, dissimilar atoms are allowed to exchange sites at nearest-neighbor distance, as long as the Metropolis criterion is met, i.e., the exchange is accepted if it lowers the energy of the cell or it is accepted with a certain probability if it does not benefit the energy balance of the system. An indicative example is shown in Fig. 2.a, which displays the final state of a Monte Carlo simulation. Unless otherwise noted, all 

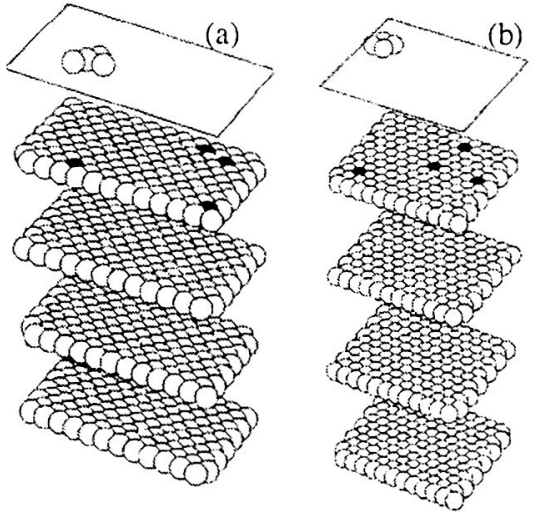

Figure 2: Final state of 4 Co atoms (black spheres) in a $\mathrm{Cu}(\mathrm{a})(100)$ and (b) (111) surface, at $\mathrm{T}=300 \mathrm{~K}$. simulations where performed at room temperature in a rigid $\mathrm{Cu}$ lattice. In all cases it is observed that the ejected $\mathrm{Cu}$ atoms diffuse on the surface layer, forming clusters. The overlayer is denoted by a rectangle above the surface layer. Clearly, the difference in coordination between the (100) and (111) surfaces is small enough to ensure that the same behavior is to be expected for a $\mathrm{Cu}(111)$ surface, as shown in Fig. 2.b.

With increasing Co coverage, the simulations show that Co atoms form patches in the surface and $1 \mathrm{~b}$ layers (Fig. 3.a-c). The formation of Co aggregates is expected, due to the immiscibility of $\mathrm{Co}$ and $\mathrm{Cu}$. The interdiffusion to subsurface (1b) layers, however, deserves further study.

These patches, with high surface energy, act as pinning sites for the $\mathrm{Cu}$ islands (Fig. 3.d), leading to the formation of bi-layer $\mathrm{Co} / \mathrm{Cu}$ islands with one of the layers immersed in the substrate surface layer. It should be noted, however, that the process of Co diffusion on the surface and insertion in the surface layer is greatly enhanced with increasing temperature, as shown in Figs. 3.e-f, which repeat the results shown in Figs. 3.c-d for $\mathrm{T}=1 \mathrm{~K}$, where Co insertion in the surface layer competes with the formation of shapeless $\mathrm{Co} / \mathrm{Cu}$ patches in the overlayer. Moreover, it is precisely the diffusion of $\mathrm{Cu}$ atoms along the edges of the forming $\mathrm{Co} / \mathrm{Cu}$ islands that determines their triangular form, as seen in Fig. 4, where an initial state characterized by a $\mathrm{Co} / \mathrm{Cu}$ island of undetermined shape evolves into nearly triangular islands.

The small difference in energy between $\mathrm{Co}(\mathrm{S})$ and $\mathrm{Co}(1 \mathrm{~b})$ substitutions, indicated in Table 2 for the case of one single $\mathrm{Co}$ atom, facilitates the interdiffusion of Co atoms in the substrate, unless alternative mechanisms are allowed that identify other energetically favored processes. Experimental results, usually available for high Co coverage, suggest the presence of bi-layer islands (i.e., two layers above the surface) which, naturally, should be preceded by the formation of single-layer islands. As long as atoms are allowed to populate only the first overlayer, the resulting islands adopt a triangular form where Co atoms cluster in the center of the island, surrounded by a $\mathrm{Cu}$ brim, as shown in Fig. 5. In general, the Co core also adopts a triangular form, inverted with respect to the overall island shape, thus maximizing the exposed $\mathrm{Cu}$ surface and therefore minimizing the surface energy of the island.
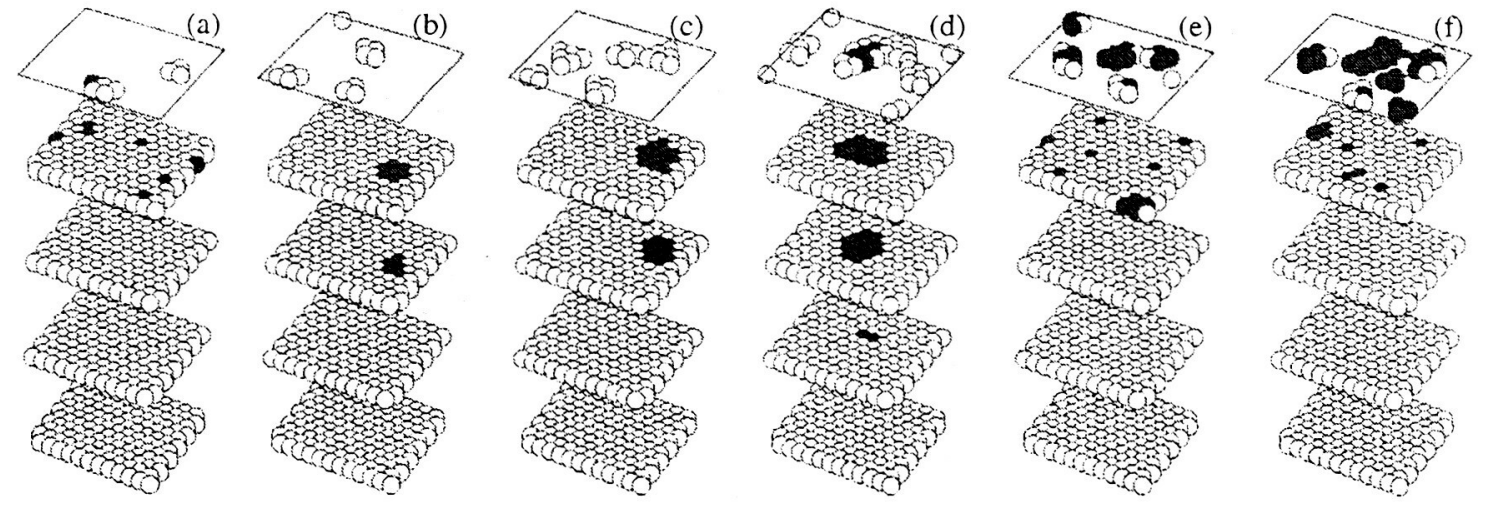

Figure 3: Final state of Monte Carlo simulations of a $\mathrm{Cu}(111)$ surface with (a) 0.056 , (b) 0.083 , (c) 0.167 , and (d) $0.250 \mathrm{ML}$ Co coverage. In every case, the initial state is given by similar cells where Co atoms are randomly dispersed on the $\mathrm{Cu}$ substrate. All simulations were performed at $\mathrm{T}=300 \mathrm{~K}$, except (e) and (f) where $\mathrm{T}=1 \mathrm{~K}$. 
ure in a rigid : ejected $\mathrm{Cu}$ lusters. The urface layer. in the (100) nat the same e, as shown ations show id 16 layers is expected, rdiffusion to er study.

$t$ as pinning le formation s immersed d, however, $r$ is greatly lts shown in ormation of of $\mathrm{Cu}$ atoms 1 , as seen in evolves into $\mathrm{d}$ in Table 2 le substrate, d processes. of bi-layer ie formation erlayer, the the island, a triangular I Cu surface

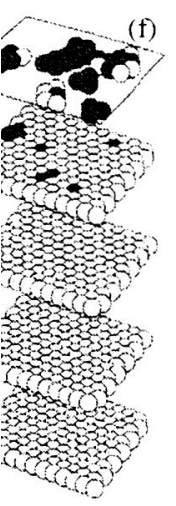

0.167 , and $(d)$ are randomly $=1 \mathrm{~K}$.
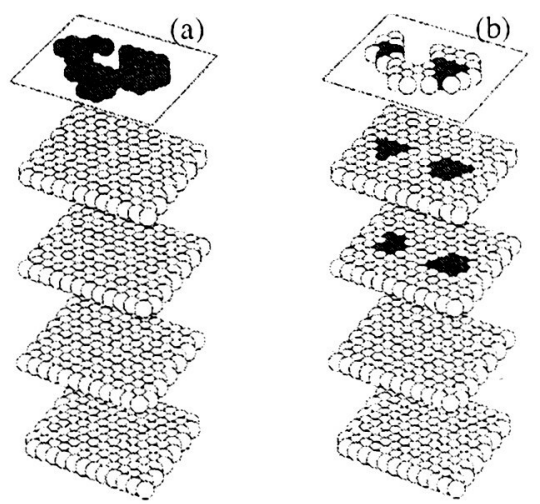

Figure 4: Initial and tinal states of a Monte Carlo simulation at room temperature, corresponding to $0.25 \mathrm{ML}$ Co coverage.
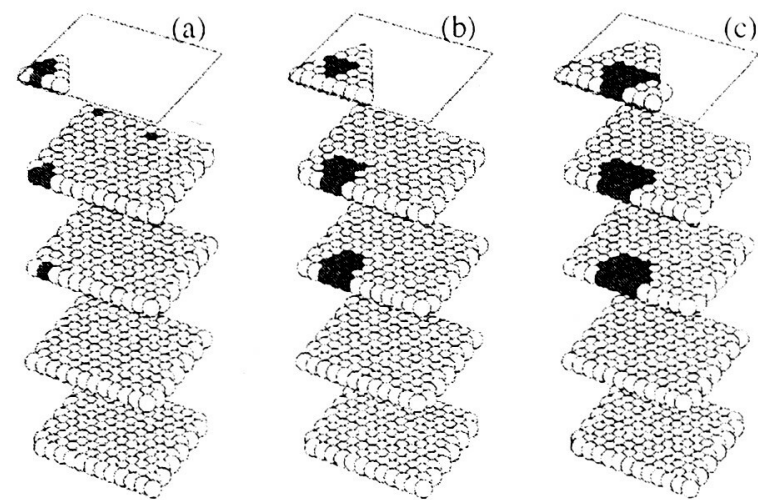

Figure 5: Final state of room temperature Monte Carlo simulations of a $\mathrm{Cu}(111$ ) surface with (a) 0.069 , (b) 0.194 , and (c) $0.313 \mathrm{ML}$ Co coverage. In every case, the initial state is given by similar cells with Co islands.

However, this is not the lowest energy configuration. A calculation showing the competition between single- and bi-layer (on the Cu(111) surface layer) islands shows that there is a maximum island size beyond which the growth of a second layer is favored. Fig. 6 shows the BFS formation energies of single- and bi-layer islands with the same number of atoms. It is seen that while the BFS chemical energy favors the bi-layer island, regardless of size, there is a noticeable gain in strain energy that drives the transition from single- to bi-layer growth beyond a certain island size. The Monte Carlo procedure used in the preceding simulations does not allow for the growth of a second layer above the surface, as exchanges of pairs of atoms are considered one at a time. However, creating an initial state with some Co atoms on the second layer suffices to trigger the corresponding process. Allowing the Monte Carlo simulations to deal with the growth of a second layer on the $\mathrm{Co} / \mathrm{Cu}$ islands leads to a similar mixed composition layer at the expense of the subsurface $\mathrm{Co}$, thus leading to tri-layer $\mathrm{Co} / \mathrm{Cu}$ islands where the first layer is immersed in the $\mathrm{Cu}$ substrate. As observed in the single layer case, the simulations show

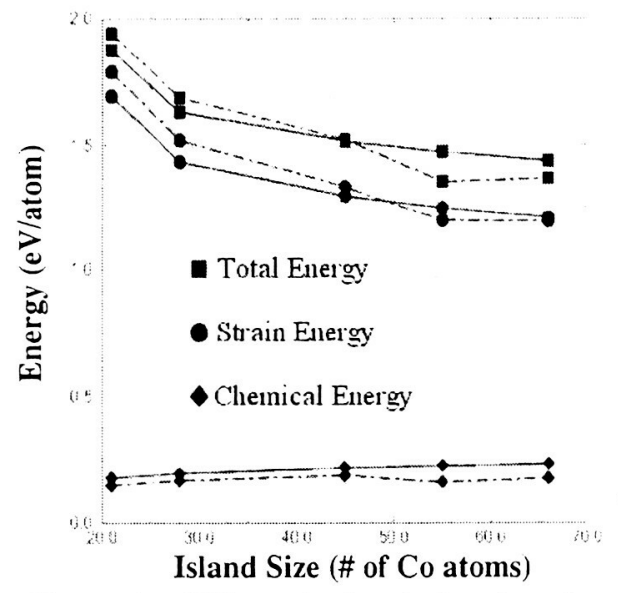

Figure 6: BFS total, chemical and strain energies for different 1 - and 2-layer islands with varying island size. Single- (bi-) layer islands are denoted with solid (dot-dashed) lines.
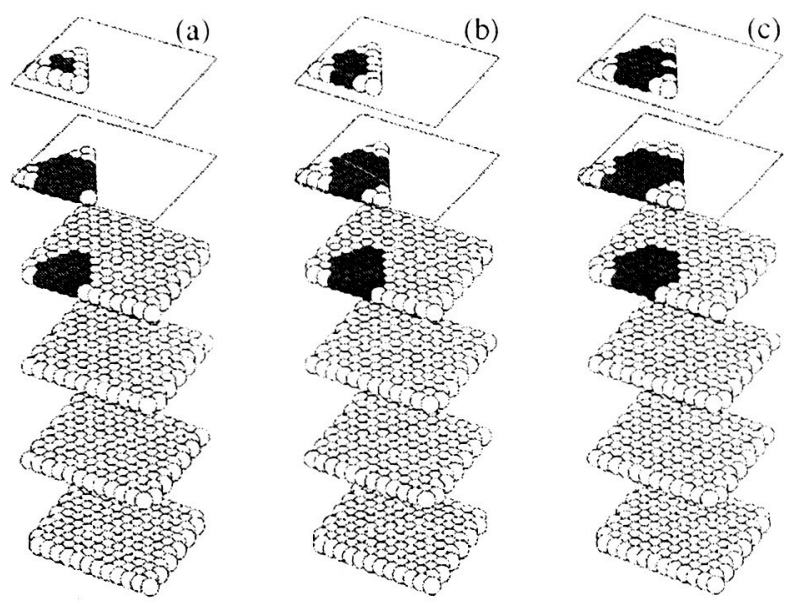

Figure 7: Final state of room temperature simulations of a $\mathrm{Cu}(111)$ surface with (a) 0.299 , (b) 0.396 , and (c) $0.507 \mathrm{ML}$ Co coverage. In every case, the initial state consists of Co islands. 
that each layer in the bi-layer islands is of mixed composition, with $\mathrm{Co}$ in the center of the island, once again exposing as much $\mathrm{Cu}$ as possible (i.e., inverted $\mathrm{Co}$ triangles), as indicated in Fig. 7. At this point, it is clear that the critical size or, correspondingly, the critical $\mathrm{Co}: \mathrm{Cu}$ ratio in the composition of the island can be reached if additional sources of $\mathrm{Cu}$ atoms - besides those replaced by Co insertions in the surface layer - are available. Fig. 8 shows the initial and final states of a simulation where additional $\mathrm{Cu}$ atoms were initially randomly distributed on the $\mathrm{Cu}(111)$ surface, thus simulating the possibility of $\mathrm{Cu}$ atoms (decoupled from nearby vacancy pools, as seen in Fig. 1) diffusing along the surface. This additional amount of Cu clearly optimizes the energy balance of the resulting island, drastically inhibiting Co interdiffusion to subsurface layers, thus achieving a final shape and structure consistent with experiment.
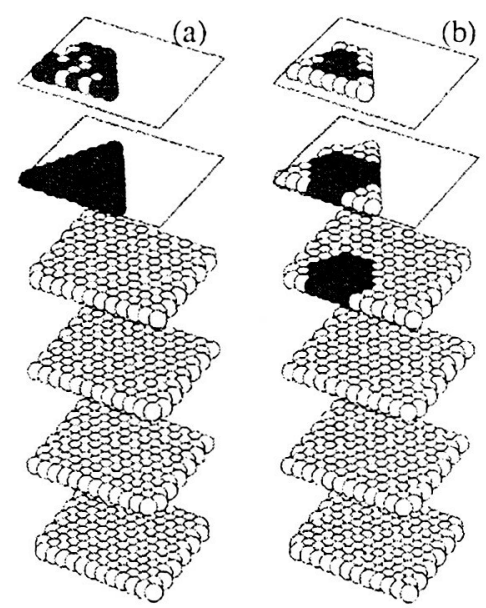

Figure 8: Initial and final states of a room temperature simulation of a $\mathrm{Cu}(111)$ surface with $0.17 \mathrm{ML}$ Co coverage.

\section{CONCLUSIONS}

The basic features of the process of island formation in the deposition of $\mathrm{Co}$ on $\mathrm{Cu}(111)$ were described within the framework of a simple modeling approach based on the BFS method for alloys: 1) intermixing of $\mathrm{Co}$ with the $\mathrm{Cu}(111)$ substrate, 2) formation of a Co surface layer on which mixed composition single- and bi-layer islands form, 3 ) evidence for a triangular island shape, 4) the existence of a critical island size, establishing the transition from a single- to a bilayer growth pattern, 5) details on the distribution of $\mathrm{Co}$ and $\mathrm{Cu}$ atoms in the different layers, both in composition and location of the different atoms, and 6) hints on the temperature dependence of the above mentioned processes. The BFS analysis of the energetics of the system allows for the identification of competing driving mechanisms for the observed behavior.

\section{ACKNOWLEDGMENTS}

Fruitful discussions with N. Bozzolo are gratefully acknowledged. This work was supported by the HOTPC project at NASA Glenn Research Center, and by the European Community under grant HPRN-CT-2000-00134.

\section{REFERENCES}

1. J. de la Figuera, J.E. Prieto, C. Ocal and R. Miranda, Surf. Sci. 307-309 (1994) 538.

2. G. Bozzolo, R.D. Noebe, J. Ferrante and C. Amador, J. Comput.-Aided Mater. Design 6 (1999) 1.

3. J. R. Smith, T. Perry, A. Banerjea, J. Ferrante and G. Bozzolo, Phys. Rev. B 44 (1991) 6444; G. Bozzolo, J. Ferrante and A. M. Rodriguez, J. Computer-Aided Mater. Design 1 (1993) 285.

4. P. Blaha, K. Schwartz and J. Luitz, WIEN97, Vienna University of Technology. Improved and updated Unix version of the copyrighted WIEN code, P. Blaha, P. Schwartz, P. Sorantin and S B Trickey, Comput. Phys. Commun. 59 (1990) 399.

5. J. de la Figuera, J. E. Prieto, C. Ocal and R. Miranda, Phys. Rev. B 47 (1993) 13(1433; Surf. Sci. 349 (1996) L139.

6. A. Rabe, N. Memmel, A. Steltenpohl and T. Fauster, Phys. Rev. Lett. 73 (1994) 2728.

7. M. Ø. Pedersen, I. A. Bönicke, E. Lagsgaard, I. Stensgaard, F. Besenbacher, A. Ruban, and J. K. Nørskov, Surf. Sci. 387 (1997) 86.

8. L. Gómez, C. Slutzky, J. Ferrón, J. de la Figuera, J. Camarero, A. L. Vázquez de Parga, J. J. de Miguel, and R. Miranda, Phys. Rev. Lett. 84 (2000) 4397. 